\title{
ON ZERO-DIMENSIONALITY AND FRAGMENTED RINGS
}

\author{
Jim Coykendall, David E. Dobbs and Bernadette Mullins
}

\begin{abstract}
A commutative ring $R$ is said to be fragmented if each nonunit of $R$ is divisible by all positive integral powers of some corresponding nonunit of $R$. It is shown that each fragmented ring which contains a nonunit non-zero-divisor has (Krull) dimension $\infty$. We consider the interplay between fragmented rings and both the atomic and the antimatter rings. After developing some results concerning idempotents and nilpotents in fragmented rings, along with some relevant examples, we use the "fragmented" and "locally fragmented" concepts to obtain new characterisations of zero-dimensional rings, von Neumann regular rings, finite products of fields, and fields.
\end{abstract}

\section{INTRODUCTION}

All rings considered below are commutative with identity. As in [6], a ring $R$ is said to be fragmented if for each nonunit $r \in R$, there exists at least one nonunit $s \in R$ such that $r \in \bigcap_{n=1}^{\infty} R s^{n}$. The theory of fragmented rings was initiated in the context of integral domains in [7], with special attention to the semi-quasilocal case and issues involving prime spectra and (Krull) dimension. The fragmented-theoretic arena was broadened to rings possibly with zero-divisors, as above, in [6], which also pursued factorisation-theoretic connections. In the present work, we first observe (Theorem 2.1) that any fragmented ring possessing a nonunit non-zero-divisor must have infinite dimension, generalising $[7$, Corollary 2.8] from the quasilocal integral domain case. Thus, a fragmented ring either is infinite-dimensional or has the property that each of its nonunits is a zero-divisor (the latter option holding for any zero-dimensional ring). Although zero-dimensional integral domains are fields (and hence do not sustain interesting ideal theory), zero-dimensional fragmented rings do relate well to several central concepts in ring theory and hence form the focus for much of this work. In particular, Section 4 uses the "fragmented" concept to develop new characterisations of zero-dimensional rings, von Neumann regular rings, finite products of fields, and fields (see Theorem 4.2 and Corollaries 4.3, 4.4 and 4.8). As a byproduct, we also find contact with connected rings (see Proposition 4.7) and

Received 25th January, 1999

This work was completed while the third-named author was on leave at the University of Tennessee, Knoxville. Thanks are expressed to UTK for its hospitality and Youngstown State University for reassigned time.

Copyright Clearance Centre, Inc. Serial-fee code: 0004-9729/99 \$A2.00+0.00. 
Noetherian rings (Corollary 4.4). The semi-quasilocal case receives emphasis in Theorem 4.9, which establishes that a semi-quasilocal ring is von Neuman regular if and only if it is zero-dimensional and fragmented. Example 4.10 shows, however, that a zerodimensional fragmented ring need not be von Neumann regular (that is, it may have a nonzero nilpotent element).

Prior to the applications mentioned above, Section 3 is devoted to an extensive study of atoms, idempotents and nilpotents in fragmented rings. We exhibit interplay between "fragmented" and factorisation-theoretic concepts such as "atomic" (in the sense of [1]) and "antimatter" (in the sense of $[5,6]$ ). For instance, Theorem 3.5 establishes that a zero-dimensional ring $R$ is fragmented if and only if each nonunit of $R$ is divisible by some nonunit idempotent of $R$; and Proposition 3.11 establishes that the zero-dimensional fragmented atomic rings are just the finite products of fields. Also noteworthy in Section 3 are examples of a fragmented ring containing a nonzero nilpotent element (in Example 3.2 ); and a fragmented integral domain with infinitely many maximal ideals (Example $3.8)$.

For a ring $A$, we denote the set of units of $A$ by $U(A)$; the set of zero-divisors of $A$ by $Z(A)$; the Jacobson radical of $A$ by $J(A)$; the nilradical of $A$ by $\operatorname{Nil}(A)$; the prime spectrum of $A$ by $\operatorname{Spec}(A)$; the Krull dimension of $A$ by $\operatorname{dim}(A)$; the set of maximal ideals of $A$ by $\operatorname{Max}(A)$; and the set of minimal ideals of $A$ by $\operatorname{Min}(A)$. It will be convenient to decree that the zero ring is zero-dimensional. Additional notation and background will be introduced as needed. Any unexplained material is standard, as in $[8,9,10]$.

\section{ThE DIMENSIONAL DICHOTOMY}

It was shown in [7, Corollary 2.8] that any quasilocal fragmented integral domain either is a field (that is, has dimension 0 ) or has dimension $\infty$. The main result of this section generalises this result by eliminating both the "quasilocal" and the "integral domain" restrictions, at the cost of adding an assumption about zero-divisors.

THEOREM 2.1. Let $R$ be a nonzero fragmented ring which contains a nonunit non-zero-divisor $x$. Then some maximal ideal $M$ of $R$ contains a strictly increasing chain $\left\{P_{n}\right\}$ of prime ideals of $R$ such that $x \in P_{n}$ for each $n$. In particular, the height of $M$ in $R$ is $\infty$, and so $\operatorname{dim}(R)=\infty$.

Proof: (Sketch) This result was established in [4, Theorem 2.3] for the case in which $R$ is an integral domain. The proof of that result and the proofs of the lemmas leading up to it carry over to the present situation because of the following observations. If $x_{1}:=x$ and $x_{n} \in \bigcap_{k=1}^{\infty} R x_{n+1}^{k}$ with $x_{n} \in R$ for each $n \geqslant 1$, then each $x_{n}$ inherits from $x$ the property of being a nonunit non-zero-divisor. In particular, any product of powers of the $x_{j}$ is also a non-zero-divisor, thus permitting the "By cancellation" step near the end of the proof of $[4$, Lemma 2.2]. 
The next result follows immediately from Theorem 2.1 .

COROLLARY 2.2. Let $R$ be a fragmented ring. Then at least one of the following conditions holds:

(i) Each nonunit of $R$ is a zero-divisor of $R$;

(ii) $\operatorname{dim}(R)=\infty$.

Any zero-dimensional ring satisfies condition (i) in Corollary 2.2 [9, Theorem 91]. We do not know of any fragmented ring which has finite positive (Krull) dimension. (Of course, any such example would satisfy condition (i).) Apart from any such rings, other fragmented rings $R$ must satisfy the dichotomy referred to in the title of the section: either $\operatorname{dim}(R)=0$ or $\operatorname{dim}(R)=\infty$.

\section{ON IDEMPOTENTS, NILPOTENTS AND ATOMS IN FRAGMENTED RINGS}

Recall from [6] that if $R$ is a ring and $r \in R \backslash U(R)$, then $r$ is said to fragment (in $R$ ) (or is said to be a fragmented element of $R$ ) if there exists $s \in R \backslash U(R)$ such that $r \in \bigcap_{n=1}^{\infty} R s^{n}$. Of course, a ring $R$ is a fragmented ring if and only if each of its nonunits is fragmented. We begin with an elementary result which collects some useful information.

Proposition 3.1 .

(a) If $e \neq 1$ is an idempotent element of a ring $R$, then $e$ fragments in $R$.

(b) A fragmented element need not be idempotent.

(c) Each multiple of a fragmented element fragments. In particular, if e $\neq 1$ is an idempotent of a ring $R$, then each element of the ideal Re fragments in $R$.

(d) A divisor of a fragmented element need not fragment.

(e) There is no logical connection between the concepts of "fragmented element of $R$ " and "nilpotent element of $R$ ".

ProOF:

(a) For each $n \geqslant 1, e=1 e^{n} \in R e^{n}$.

(b) In $R=\mathbf{Z} / 12 \mathbf{Z}, r:=3+12 \mathbf{Z}$ is a nonunit which fragments since $r \in \bigcap_{n=1}^{\infty} R r^{n}$. Of course, $r$ is not idempotent because $9 \not \equiv 3(\bmod 12)$. (In this example, $R$ does have nontrivial idempotents, namely $4+12 \mathrm{Z}$ and $9+12 \mathrm{Z}$, which fragment by (a).)

(c) If $r, s \in R$ satisfy $r \in \bigcap_{n=1}^{\infty} R s^{n}$ and $t \in R$, then $t r \in R r \subseteq \bigcap_{n=1}^{\infty} R s^{n}$.

(d) In $R=\mathbf{Z} / 12 \mathrm{Z}, 6+12 \mathbf{Z}$ fragments (either by direct calculation or by combining (b) and (c)), although its divisor $2+12 Z$ does not fragment in $R$. (The last assertion can be verified here by exhaustive calculation. A quicker, more conceptual proof is available using the "very stong atom" idea which appears later in this section.) 
(e) In the ring $\mathbf{Z} / 12 Z, 3+12 \mathbf{Z}$ is a fragmented element which is not nilpotent, $2+12 \mathbf{Z}$ is a non-fragmented nonunit which is not nilpotent, and $6+12 \mathrm{Z}$ is a fragmented element which is nilpotent; and $2+4 \mathrm{Z}$ is a non-fragmented nonunit which is nilpotent in the ring $\mathrm{Z} / 4 \mathrm{Z}$.

In view of Proposition 3.1 (e), it seems reasonable to ask whether a fragmented ring can contain a nonzero nilpotent element. The answer is in the negative for rings of the form $\mathbf{Z} / n \mathbf{Z}$. Indeed, by [6, Proposition 17$], \mathbf{Z} / n \mathbf{Z}$ is fragmented if and only if $n$ is a product of pairwise distinct primes, in which case $\mathbf{Z} / n \mathbf{Z}$ is isomorphic to a product of fields and hence contains no nonzero nilpotents. Nevertheless, Example 3.2 answers the general question in the affirmative. A zero-dimensional example to the same effect appears in Example 4.10 below.

EXAmPLE 3.2. There exists a fragmented ring which contains a nonzero nilpotent element.

Proof: Consider a valuation domain $V$ (constructed, for instance, as in $[\mathbf{1 1}$, Corollary 3.6]) whose prime spectrum is the countable set $\left\{P_{n}: n \geqslant 0\right\} \cup\{M\}$, where

$$
0=P_{0} \subset P_{1} \subset \cdots \subset P_{n} \subset \cdots \subset M .
$$

Choose $x \in P_{1} \backslash P_{0}$, and set $R:=V /\left(x^{2}\right)$. We claim that $R$ has the asserted properties. First, note that $V$ is fragmented, by [7, Corollary 2.6], since $M$ is unbranched in $V$. As $\left(x^{2}\right) \subseteq M=J(V)$, it follows from [6, p.223] that $R$ is also fragmented. Moreover, $x+\left(x^{2}\right) \in R$ is nilpotent (since its square is 0 ) and nonzero (since $x$ is a nonunit of $V$ ). This establishes the claim and completes the proof. (Observe that $\operatorname{dim}(R)=\infty$, and so this example does not shed light on the question of whether there exists a fragmented ring of finite positive dimension which satisfies condition (i) in Corollary 2.2.)

We begin our focus on the zero-dimensional case with the following straightforward result.

Proposition 3.3. Let $R$ be a zero-dimensional ring. Then:

(a) For each $r \in R$, there exists $n \geqslant 1$ such that $r^{n}=u e$ for some $u \in U(R)$ and some idempotent $e \in R$.

(b) For each $r \in R \backslash U(R)$, there exists $n \geqslant 1$ such that $r^{n}$ fragments in $R$.

(c) If $R$ is nonzero and not quasilocal, then some nonzero element of $R$ fragments in $R$.

PROOF:

(a) According to [8, Theorem 3.2], the conclusion characterises $\pi$-regularity in the class of total quotient rings. The conclusion follows since any zero-dimensional ring is a $\pi$-regular total quotient ring (see [8, Theorem 3.1]).

(b) Combine (a) with Proposition 3.1 (a), (c). 
(c) The hypothesis allow us to choose $M \in \operatorname{Max}(R)$ and to conclude that $\mathrm{Nil}(R) \subset$ $M$. Pick $r \in M \backslash \operatorname{Nil}(R)$. By (b), $r^{n}$ fragments for some $n \geqslant 1$; moreover, $r^{n} \neq 0$ since $r$ is not nilpotent.

We next show that, in some sense, parts (b) and (c) of Proposition 3.3 are best possible.

REMARK 3.4. (a) One may verify that $R:=\mathrm{Z} / 12 \mathrm{Z}$ is an example of a zero-dimensional ring in which $r^{2}$ fragments for every $r \in R \backslash U(R)$, although [6, Proposition 17] shows that $R$ is not a fragmented ring since 12 is not a square-free integer. A more trivial example of this phenomenon is provided by the ring $\mathrm{Z} / 4 \mathrm{Z}$.

(b) Proposition 3.3 (c) fails without the hypothesis that $R$ is not quasilocal. To see this, suppose that a nonzero ring $R$ is zero-dimensional and quasilocal. Then $R$ has a unique prime ideal, and so each nonunit of $R$ is nilpotent. It follows easily that no nonzero element of $R$ is fragmented.

Continuing the theme in Proposition 3.3 (a), we next characterise fragmented rings in the class of zero-dimensional rings.

THEOREM 3.5. Let $R$ be a zero-dimensional ring. Then the following conditions are equivalent:

(1) If $r \in R \backslash U(R)$, then $r$ is divisible by some idempotent $e \neq 1$ of $R$.

(2) $R$ is a fragmented ring.

PROOF:

(1) $\Rightarrow(2)$ : We show that each $r \in R \backslash U(R)$ fragments. By (1), $r=$ se for some $s \in R$ and some idempotent $e \neq 1$ of $R$. The conclusion now follows by combining parts (a) and (c) of Proposition 3.1.

$(2) \Rightarrow(1)$ : Let $r \in R \backslash U(R)$. By (2), $r \in \bigcap_{n=1}^{\infty} R s^{n}$ for some $s \in R \backslash U(R)$. By Proposition $3.3(\mathrm{a})$, there exists $m \geqslant 1$ such that $s^{m=1}=u e$ for some $u \in U(R)$ and some idempotent $e \in R$. As $s$ is a nonunit of $R$, so is $e$; that is, $e \neq 1$. Since $r=t s^{m}$ for some $t \in R$, we have that $r=(t u) e$ is divisible in $R$ by $e$.

Our focus now sharpens to the context of von Neumann regular rings. Recall that a ring $R$ is said to be von Neumann regular if for each $a \in R$, there exists $b$ in $R$ such that $a=a^{2} b$; equivalently, if $R_{M}$ is a field for each $M \in \operatorname{Max}(R)$ (see [2, Exercise 9, page 138]). Some other characterisations of von Neumann regularity are more obviously germane to the present work. Specifically, a ring $R$ is von Neumann regular if and only if each principal ideal of $R$ can be generated by an idempotent; equivalently, if each $r \in R$ can be expressed in the form $r=u e$ for some $u \in U(R)$ and some idempotent $e$ of $R$. Most telling for our purposes is the following characterisation: a ring $R$ is von Neumann regular if and only if $R$ is zero-dimensional and reduced (see [2, Exercise 16(d), p.143]).

Proposition 3.6. Any von Neumann regular ring is a fragmented ring. 
Proof: Let $R$ be a von Neumann regular ring. By the above remarks, $\operatorname{dim}(R)=0$. Accordingly, by Theorem 3.5, it suffices to show that if $r \in R \backslash U(R)$, then $r$ is divisible by some idempotent $e \neq 1$ of $R$. Now, by von Neumann regularity, the principal ideal $R r=R f$ for some idempotent $f$ of $R$. Since $r$ is a nonunit of $R$, so is $f$; and, of course, $r$ is divisible by $f$ in $R$.

The next result provides other useful examples of fragmented rings.

PROPOSITION 3.7. Let a ring $R$ be ring-isomorphic to $\Pi R_{\alpha}$, a nonempty product of rings $R_{\alpha}$. Then $R$ is a fragmented ring if and only if $R_{\alpha}$ is a fragmented ring for each $\alpha$.

Proof: We first prove the "only if" assertion. Without loss of generality, $R=$ $R_{1} \times R_{2}$ and we need only show that $R_{1}$ is fragmented. Let $c \in R_{1} \backslash U\left(R_{1}\right)$. Since $R$ is fragmented by assumption, $r:=(c, 1) \in \bigcap_{n=1}^{\infty} R(a, b)^{n}$ for some $(a, b) \in R \backslash U(R)$. It follows that $b \in U\left(R_{2}\right)$ and $a \in R_{1} \backslash U\left(R_{1}\right)$. As $c \in \bigcap_{n=1}^{\infty} R_{1} a^{n}, c$ fragments in $R_{1}$, as desired.

For the "if" assertion, we may suppose that $R=\Pi R_{\alpha}$, where each $R_{\alpha}$ is fragmented. Let $r=\left(r_{\alpha}\right) \in R \backslash U(R)$. Consider an index $\alpha$. If $r_{\alpha} \in U\left(R_{\alpha}\right)$, put $s_{\alpha}:=1 \in R_{\alpha}$. On the other hand, if $r_{\alpha} \in R_{\alpha} \backslash U\left(R_{\alpha}\right)$, choose $s_{\alpha} \in R_{\alpha} \backslash U\left(R_{\alpha}\right)$ such that $r_{\alpha} \in \bigcap_{n=1}^{\infty} R_{\alpha} s_{\alpha}^{n}$. Put $s:=\left(s_{\alpha}\right) \in R$. Of course, $r \in \bigcap_{n=1}^{\infty} R s^{n}$. Moreover, $s \in R \backslash U(R)$ because there is an index $\alpha$ such that $s_{\alpha} \in R_{\alpha} \backslash U\left(R_{\alpha}\right)$ (since the assumption on $r$ ensures that there is an index $\alpha$ such that $\left.r_{\alpha} \in R_{\alpha} \backslash U\left(R_{\alpha}\right)\right)$.

Since any field is evidently a fragmented ring, we see (by using an infinite index set in Proposition 3.7) that a fragmented ring need not be semi-quasilocal. The corresponding question for integral domains has not been addressed in the earlier literature. Example 3.8 settles this issue.

EXAMPLE 3.8. There exists a fragmented integral domain with infinitely many maximal ideals.

ProOF: An integral domain $R$ with the asserted properties can be constructed as follows. First, let $A:=\mathbf{F}_{2}\left[\left\{x_{i, j},\left(x_{i, j} / x_{i, j+1}^{n}\right): i \geqslant 1, j \geqslant 1, n \geqslant 1\right\}\right]$. Consider the collection $\left\{P_{i}: i \geqslant 1\right\} \subseteq \operatorname{Spec}(A)$, where $P_{i}=\left(\left\{x_{i, j},\left(x_{i, j} / x_{i, j+1}^{n}\right): j \geqslant 1, n \geqslant 1\right\}\right)$. Then $S:=A \backslash \bigcup_{i=1}^{\infty} P_{i}$ is a multiplicatively closed subset of $A$; set $R:=A_{S}$.

We claim that $R$ is fragmented. Observe that each $x_{i, j}$ fragments in $R$ since $x_{i, j} \in$ $\bigcap_{n=1}^{\infty} R x_{i, j+1}^{n}$. Thus, to prove the claim, it suffices, by Proposition 3.1 (c), to show that each nonunit of $R$ is a multiple of some $x_{i, j}$. Let $y \in R \backslash U(R)$. Since $y=a / s$ for some $a \in A \backslash S$ and some $s \in S$, it suffices to show that $a$ is a multiple of some $x_{i, j}$ in $R$. As $a \notin S=A \backslash \bigcup_{i=1}^{\infty} P_{i}$, we have that $a$ lies in some $P_{i}$. Without loss of generality, $a \in P_{1}$. Then $a$ can be expressed in the form $x_{1,1} f_{1}+\cdots+x_{1, s} f_{s}+\left(x_{1,1} / x_{1,2}^{n_{1}}\right) g_{1}+\cdots+\left(x_{1, t} / x_{1, t+1}^{n_{t}}\right) g_{t}$ 
for some nonnegative integers $s$ and $t$ and some $f_{i}, g_{j} \in A$. Let $m=1+\max \{s, t\}$. Note that $x_{1, m}$ divides $x_{1, j}$ for any $j \leqslant m$ and also that $x_{1, m}$ divides $x_{1, j} / x_{1, j+1}$ for any $j<m$. It follows that $x_{1, m}$ divides each term of $a$, and hence, $x_{1, m}$ divides $a$ in $A$. Thus, $a$ is a multiple in $R$ of a fragmented element, to complete the proof of the claim.

We show $\operatorname{Max}(R)$ is infinite. For each $i \geqslant 1$, observe that $x_{i, 1}$ is a nonunit of $R$, and so must lie in a maximal ideal, say $M_{i}$, of $R$. Suppose $M_{i}=M_{i^{\prime}}$, with $i \neq i^{\prime}$. Then $z:=x_{i, 1}+x_{i^{\prime}, 1} \in M_{i}$. Viewing $z \in A$, note that $z \notin P_{i} \cup P_{i^{\prime}}$. Suppose $z \in P_{k}$. Expand $z$ after expressing it as a linear combination of monomials in the $x_{k, j},\left(x_{k, j} / x_{k, j+1}^{n}\right)$, and let $\lambda$ be minimal such that $x_{k, \lambda}$ appears in a numerator. Degree $x_{x_{k, \lambda}}$-considerations lead to a contradiction; so no such $k$ exists. Hence, $z \in S=A \backslash \bigcup_{i=1}^{\infty} P_{i} \subseteq U(R)$, a contradiction. Thus, the $M_{i}$ are distinct.

For the final theme of this section, we make contact with some of the factorisationtheoretic concepts from $[1,5,6]$. Recall that if $R$ is a ring, then $a, b \in R$ are said to be very strong associates (or $a$ is very strongly associated to $b$ ) if either (i) $a=b=0$ or (ii) $a \neq 0, R a=R b$, and $r \in U(R)$ for all $r \in R$ such that $a=r b$. Also, a nonunit $a \in R$ is said to be a very strong atom if $a=b c$ with $b, c \in R$ implies $a$ is very strongly associated with either $b$ or $c$. It was shown in [1] that the concept of a "very strong atom" in a ring possibly with zero-divisors is closely related to the familiar concept of an "atom" (also called an "irreducible element") in an integral domain. Indeed, a nonzero nonunit $a$ in a ring $R$ is a very strong atom of $R$ if and only if $a=b c$ with $b, c \in R$ implies either $b$ or $c$ is a unit of $R$ [1, Theorem 2.5].

Moreover, an integral domain $R$ is called an antimatter domain if it does not contain any atoms. Similarly, a ring $R$ is called an antimatter ring if $R$ does not contain any very strong atoms except possibly zero. It was noted in [5] that any fragmented integral domain is an antimatter integral domain. The next proposition generalises this result to the context of arbitrary commutative rings.

\section{PROPOSITION 3.9. Any fragmented ring is an antimatter ring.}

Proof: Deny. Choose a fragmented ring $R$ which is not an antimatter ring. Then there exists a nonzero very strong atom $a \in R$. Since $a \in R \backslash U(R)$ and $R$ is fragmented, there exists $s \in R \backslash U(R)$ such that $a \in \bigcap_{n=1}^{\infty} R s^{n}$. In particular, $a=r s^{2}$ for some $r \in R$. But then $a=(r s) s$, where neither $r s$ nor $s$ is a unit, contradicting the above-mentioned criterion that $a$ be a very strong atom of $R$.

In addition to the concept of a "very strong atom," other related concepts have been employed in the literature to study factorisation in commutative rings. Following [1], a nonunit $a$ in a ring $R$ is said to be an m-atom of $R$ if $R a$ is maximal in the set of proper principal ideals of $R$. Also recall that if $R$ is a ring, then $a, b \in R$ are said to be strong associates (respectively, associates) if $a=b u$ for some $u \in U(R)$ (respectively, $R a=R b$ ). Then a nonunit $a \in R$ is said to be a strong atom (respectively, atom) if $a=b c$ with 
$b, c \in R$ implies $a$ is strongly associated (respectively, associated) with either $b$ or $c$. The various types of "atoms" discussed above are related in general by the following nonreversible implications: nonzero very strong atom $\Rightarrow m$-atom $\Rightarrow$ strong atom $\Rightarrow$ atom [1]. It should be noted, however, that these four conditions are equivalent in the setting of integral domains.

We further recall that a ring $R$ is said to be very strongly atomic (respectively, $m$ atomic; respectively, strongly atomic; respectively, atomic) if every nonzero nonunit of $R$ is a finite product of very strong atoms (respectively, $m$-atoms; respectively, strong atoms; respectively, atoms). Note that the zero ring vacuously satisfies the very strongly atomic condition. Also recall that for an integral domain, Noetherian implies atomic. This implication holds more generally for a ring possibly with zero-divisors (see $[1$, Theorem $3.2]$ ). Nevertheless, a Noetherian ring need not be very strongly atomic, as can be seen by applying the criterion in [6, Corollary 13$]$ to $\mathrm{Z} / 12 \mathrm{Z}$.

We also say that a ring $R$ is an antimatter (respectively, $m$-antimatter; respectively, highly antimatter; respectively, very highly antimatter) ring if $R$ does not contain any very strong atoms (respectively, $m$ - atoms; respectively, strong atoms; respectively, atoms) except possibly zero. It is easily verfied that very strongly atomic $\Rightarrow m$-atomic $\Rightarrow$ strongly atomic $\Rightarrow$ atomic; and that very highly antimatter $\Rightarrow$ highly antimatter $\Rightarrow$ $m$-antimatter $\Rightarrow$ antimatter. Although Proposition 3.9 showed that a fragmented ring cannot contain any nonzero very strong atoms, we show in Remark 3.10 (a) that it is possible for such a ring to contain a nonzero $m$-atom.

REMARK 3.10. (a) A fragmented ring $R$ need not be $m$-antimatter (and therefore, $R$ need not be highly antimatter or very highly antimatter). For an example, note that $R:=\mathrm{Z} / 6 \mathbf{Z}$ is a fragmented ring by Proposition 3.7 (or the criterion in [6, Proposition $17]$ ), but $R$ is not $m$-antimatter since $2+6 \mathbf{Z}$ and $3+6 \mathbf{Z}$ are evidently $m$-atoms of $R$.

(b) Let $R$ be a zero-dimensional fragmented ring. As announced above, Example 4.10 shows that $R$ need not be reduced; that is, $R$ need not be von Neumann regular. Equivalently, it need not be the case that each principal ideal of $R$ can be generated by an idempotent. We next observe, however, that at least some important principal ideals of $R$ are so generated.

Specifically, we claim that if $R$ is a zero-dimensional fragmented ring and $r \in R$ is an $m$-atom, then there exists an idempotent $e$ of $R$ such that $R r=R e$. For a proof, note that since $r \in R \backslash U(R)$, Theorem 3.5 ensures that $r$ is divisible by some idempotent $f \neq 1$ of $R$. Thus $R r \subseteq R f \subset R$, and hence by the maximality of $R r$, it follows that $R r=R f$, as desired.

The next result combines the "zero-dimensional fragmented" and "atomic" themes to produce a characterisation of an important class of von Neumnann regular rings. We first collect three useful results from [1]. First, let $R=\Pi R_{\alpha}$ be a nonempty product of rings $R_{\alpha}$. Then an element $\left(r_{\alpha}\right) \in R$ is an atom of $R$ if and only if there exists an index 
$\alpha_{0}$ such that $r_{\alpha_{0}}$ is an atom of $R_{\alpha_{0}}$ and $r_{\alpha} \in U\left(R_{\alpha}\right)$ for all $\alpha \neq \alpha_{0}$ [1, Theorem 2.15(2)]. Secondly, a nonempty product $R=\Pi R_{\alpha}$ of rings $R_{\alpha}$ is atomic if and only if the index set is finite and $R_{\alpha}$ is atomic for each $\alpha$ [1, Theorem 3.4(1)]. Finally, if a ring $R$ is atomic, then $R$ is a finite direct product of indecomposable atomic rings [1, Corollary 3.5].

Propos ITION 3.11. Let $R$ be a ring. Then the following conditions are equivalent:

(1) $R$ is a zero-dimensional fragmented atomic ring;

(2) $R$ is a product of finitely many fields (with an empty product being viewed as the zero ring).

Proof: $(2) \Rightarrow(1)$ : Assume (2). Then $R$ is evidently zero-dimensional; $R$ is fragmented by Proposition 3.7; and $R$ is atomic by a result recalled above [1, Theorem $3.4(1)]$.

$(1) \Rightarrow(2)$ : Assume (1). Since $R$ is atomic, a result recalled above [1, Corollary 3.5] implies that $R=\prod_{i=1}^{m} R_{i}$ is a finite direct product of indecomposable atomic rings $R_{i}$. As $R$ is fragmented by hypothesis, each $R_{i}$ is fragmented by Proposition 3.7. Then for each $i, R_{i}$ inherits zero-dimensionality from $R$ and, by Theorem 3.5, each nonzero nonunit of $R_{i}$ is divisible by a nontrivial idempotent of $R_{i}$. However, since $R_{i}$ is indecomposable, $R_{i}$ contains no nontrivial idempotents. Thus, each $R_{i}$ must be a field, as desired.

Observe that each of the fragmented rings characterised in Proposition 3.11 is zerodimensional and reduced, hence von Neumann regular. However, not every von Neumann regular ring satisfies the equivalent conditions in Proposition 3.11, since a product of infinitely many fields is not atomic.

\section{FRAGMENTED-THEORETIC CHARACTERISATIONS OF SOME CLASSES OF ZERO-DIMENSIONAL RINGS}

We have seen in Proposition 3.11 that the "fragmented" concept can be used to characterise finite products of fields. Corollary 4.4 will provide another fragmentedtheoretic characterisation of such rings. In fact, this section is primarily devoted to similar characterisations of some other important classes of zero-dimensional rings. We begin with a result which is valid in arbitrary dimensions. Recall that distinct prime ideals $P$ and $Q$ of a ring $A$ are said to be adjacent (in $A$ ) if one of $P, Q$ is contained in the other and no prime ideal of $A$ is contained properly between $P$ and $Q$.

Lemma 4.1. If $Q \subset P$ are adjacent prime ideals of a ring $R$, then $R_{P}$ is not a fragmented ring.

Proof: Deny. Then $A:=R_{P} / Q R_{P}$ is a fragmented ring by [6, p.223], since $Q R_{P} \subseteq$ $J\left(R_{P}\right)$. Moreover, the "adjacent" hypothesis guarantees that $\operatorname{dim}(A)=1$; and, of course, $A$ is a quasilocal integral domain. However, according to [7, Corollary 2.8], any quasilocal 
fragmented integral domain which is not a field must be infinite-dimensional, and so we have the desired contraction.

THEOREM 4.2. Let $R$ be a ring. Then the following conditions are equivalent:

(1) $R_{P}$ is a fragmented ring for each $P \in \operatorname{Spec}(R) \backslash \operatorname{Min}(R)$;

(2) $\operatorname{dim}(R)=0$.

Proof: $(2) \Rightarrow(1)$ vacuously. It suffices to establish the contrapositive of $(1) \Rightarrow(2)$. Suppose that $\operatorname{dim}(R)>0$, with $P \subset N$ distinct prime ideals of $R$. By [9, Theorem 11], we have $P \subseteq Q_{1} \subset Q_{2} \subseteq N$, for some adjacent prime ideals $Q_{1} \subset Q_{2}$ of $R$. By Lemma 4.1, $R_{Q_{2}}$ is not fragmented, although $Q_{2} \in \operatorname{Spec}(R) \backslash \operatorname{Min}(R)$.

Corollary 4.3. Let $R$ be a ring. Then $R_{P}$ is a fragmented ring for each $P \in \operatorname{Spec}(R)$ if and only if $R$ is von Neumann regular.

Proof: The "if" assertion is immediate since any von Neumann regular ring is locally a field (see [2, Exercise 9, p.138]). Conversely, suppose that $R_{P}$ is fragmented for each $P \in \operatorname{Spec}(R)$. By Corollary $4.2, \operatorname{dim}(R)=0$. Hence, it suffices to show that $R$ is reduced. Therefore, it is enough to prove that $A:=R_{P}$ is reduced for each $P \in \operatorname{Spec}(R)$. Since $A$ is zero-dimensional and quasilocal, its unique prime (maximal) ideal is $\operatorname{Nil}(A)$, and so each nonunit of $A$ is nilpotent. Now, suppose that $a \in A$ is nilpotent. Since $a \in A \backslash U(A)$ and $A$ is a fragmented ring (by hypothesis), there exists $b \in A \backslash U(A)$ such that $a \in \bigcap_{n=1}^{\infty} A b^{n}$. By the above remarks, $b$ is nilpotent and so $b^{n}=0$ for some $n \geqslant 1$, whence $a=0$ and so $A$ is reduced.

Before we pass to some fragmented rings possibly having Krull dimension 1, Corollary 4.4 characterises the zero-dimensional Noetherian fragmented rings. These form a subclass of the rings characterised in Corollary 4.3. Since any Noetherian ring is atomic, one has an alternate proof of Corollary 4.4 via an application of Proposition 3.11. The following proof avoids factorisation-theoretic ideas.

Corollary 4.4. Let $R$ be a ring. Then the following conditions are equivalent:

(1) $R$ is a zero-dimensional Noetherian fragmented ring;

(2) $R$ is a product of finitely many fields (with an empty product being viewed as the zero ring).

Proof: $(2) \Rightarrow(1)$ : Assume (2). Since any field is fragmented, $R$ is fragmented by Proposition 3.7. Then (1) follows since any finite product of zero-dimensional (respectively, Noetherian) rings is zero-dimensional (respectively, Noetherian).

$(1) \Rightarrow(2)$ : Assume (1). As $R$ is Noetherian and $\operatorname{dim}(R)=0, R$ is an Artinian ring. By the fundamental structure theorem for such rings $[13$, Theorem $3, \mathrm{p} .205], R=\Pi_{\alpha} R_{\alpha}$ for some indexed family $\left\{R_{\alpha}\right\}$ of Artin local rings $R_{\alpha}$. For each $\alpha, R_{\alpha}$ is fragmented by Proposition 3.7 and zero-dimensional (because Artinian), and so $R_{\alpha}$ is von Neumann 
regular by Corollary 4.3. Therefore, since $R_{\alpha}$ is canonically the localisation at its maximal ideal, $R_{\alpha}$ is a field.

COROLLARY 4.5. Let $R$ be a ring. Then the following conditions are equivalent:

(1) $R_{P}$ is a fragmented ring for each $P \in \operatorname{Spec}(R) \backslash \operatorname{Max}(R)$;

(2) $\operatorname{dim}(R) \leqslant 1$ and $R_{P}$ is a field for each $P \in \operatorname{Min}(R) \backslash \operatorname{Max}(R)$.

ProOf: $(2) \Rightarrow(1)$ : Trivial, since any field is fragmented.

$(1) \Rightarrow(2)$ : Assume (1). If $\operatorname{dim}(R)>1$, choose distinct prime ideals $Q_{0} \subset Q \subset N$ of $R$. By $\left[9\right.$, Theorem 11], we have $Q_{0} \subseteq Q_{1} \subset Q_{2} \subseteq Q$, for some adjacent prime ideals $Q_{1} \subset$ $Q_{2}$ of $R$. By Lemma 4.1, $R_{Q_{2}}$ is not fragmented, although $Q_{2} \in \operatorname{Spec}(R) \backslash \operatorname{Max}(R)$, a contradiction. Therefore, $\operatorname{dim}(R) \leqslant 1$. Next, consider $P \in \operatorname{Min}(R) \backslash \operatorname{Max}(R)$. Then $R_{P}$ is zero-dimensional, quasilocal and fragmented, hence von Neumann regular by Corollary 4.3 , and hence a field.

Since the localisation of an integral domain $D$ at its unique minimal prime ideal is the quotient field of $R$, the next result is a special case of Corollary 4.5.

COROLlary 4.6. Let $R$ be an integral domain. Then the following conditions are equivalent:

(1) $\quad R_{P}$ is a fragmented ring for each $P \in \operatorname{Spec}(R) \backslash \operatorname{Max}(R)$;

(2) $\operatorname{dim}(R) \leqslant 1$.

We next merge the "idempotent" theme from Section 3 with the topological aspect of the "Spec" theme introduced above. Recall that a ring $A$ is called a connected ring if and only if 0 and 1 are the only idempotent elements of $A$; equivalently, if and only if $\operatorname{Spec}(A)$ (in the Zariski topology) is a connected space [2, Corollary 2, p.104].

PROPOSITION 4.7. Let $R$ be a ring. Then the following conditions are equivalent:

(1) $\operatorname{dim}(R)=0$ and $R$ is a nonzero connected ring;

(2) $R$ has a unique prime ideal;

(3) $\operatorname{Nil}(R)=R \backslash U(R)$.

Proof: (1) $\Rightarrow(3)$ : Assume (1). Since $R$ is nonzero, each nilpotent element of $R$ is a nonunit. It remains to show that each nonunit $r \in R$ is nilpotent. As $\operatorname{dim}(R)=0$, it follows from Proposition 3.3 (a) that there exist $n \geqslant 1, u \in U(R)$ and an idempotent $e$ of $R$ such that $r^{n}=u e$. Of course, $e \neq 1$ since $r$ is a nonunit, and so $e=0$ since $R$ is connected. Therefore, $r^{n}$ is a multiple of 0 , and so $r$ is nilpotent.

(3) $\Rightarrow(2)$ : Assume (3). Let $M \in \operatorname{Max}(R)$ and $P \in \operatorname{Spec}(R)$. If there exists $r \in M \backslash P$, then $r \in(R \backslash U(R)) \backslash N$ il $(R)$, contrary to (3). Therefore, $M \subseteq P$, and (2) follows.

$(2) \Rightarrow(1)$ : Assume (2). Evidently, $\operatorname{dim}(R)=0$. Also, $\operatorname{Spec}(R)$ is a connected topological space (since its underlying set is singleton) and so, by the above remarks, $R$ 
is a connected ring. Finally, $R$ is nonzero since $\operatorname{Spec}(R)$ is nonempty.

COROLlaRY 4.8. Let $R$ be a ring. Then the following conditions are equivalent:

(1) $R$ is a nonzero zero-dimensional connected fragmented ring;

(2) $R$ is a field.

Proof: It is clear that $(2) \Rightarrow(1)$. Moreover, Proposition 4.7 and Corollary 4.3 combine easily to yield $(1) \Rightarrow(2)$.

In view of Section 3 and the earlier material in this section, it seems reasonable to ask for conditions guaranteeing that a zero-dimensional fragmented ring be von Neumann regular. Of course, Proposition 3.6 ensures that any von Neumann regular ring is fragmented (and it is surely also zero-dimensional). Thus, one should ask which zerodimensional fragmented rings must be reduced. In Theorem 4.9, we settle this in the semi-quasilocal case. First, we indicate a role for Corollary 4.8 in an analysis of the general case.

According to [12, Theorem 4.4], any ring $R$ can be realised as the ring of global sections of a suitable sheaf of connected rings defined on a Boolean space $X(R)$. In fact, $X(R)$ can be taken to be $\operatorname{Spec}(B(R))$ in the Zariski topology, where $B(R)$ denotes the Boolean ring formed by the set of idempotents of $R .(B(R)$ inherits its multiplication operation from $R$, but addition in $B(R)$ is redefined by $e \oplus f=e+f-2 e f$ for all idempotents $e, f \in R$.) Recall also that if $x \in B(R)$, then the stalk of the abovementioned sheaf at $x$ is the connected ring $R / x R$. Hence, by standard sheaf theory, $R$ is canonically isomorphic to a subring of $\prod_{x \in X(R)} R / x R$. Now, suppose that $R$ is a zero-dimensional fragmented ring. For $R$ to be reduced, it would suffice to show that $R / x R$ is reduced for each $x \in X(R)$. Evidently, $\operatorname{dim}(R / x R)=0$ for each $x \in X(R)$. Therefore, in view of Corollary 4.8 , it would suffice to show that $R / x R$ is fragmented for each $x \in X(R)$. The ring in Example 4.10 shows that $R / x R$ need not be fragmented for all $x \in X(R)$. Thus, despite [7, Lemma 2.3] and [6, p.223], an arbitrary homomorphic image of a fragmented ring need not be fragmented, even in the zero-dimensional case.

We next give a companion for Corollary 4.3.

THEOREM 4.9. Let $R$ be a semi-quasilocal ring. Then the following conditions are equivalent:

(1) $\operatorname{dim}(R)=0$ and $R$ is a fragmented ring;

(2) $R$ is von Neumann regular.

Proof: $(2) \Rightarrow$ (1) (even if $R$ is not semi-quasilocal) by Proposition 3.6. Conversely, assume (1). Without loss of generality, $R \neq 0$. Let $M_{1}, \ldots, M_{n}$ be all the (pairwise distinct) maximal ideals of $R$. We proceed by strong induction on $n$. The assertion for the case $n=1$ is an easy consequence of Corollary 4.3. Assume $n \geqslant 2$. By the Prime Avoidance Lemma [9, Theorem 81], there exists $r \in M_{1} \backslash \bigcup_{j=2}^{n} M_{j}$. Observe that the 
reduced ring associated to $R$, namely $A:=R / N i l(R)$, is zero-dimensional and reduced, hence von Neumann regular. Therefore, the principal ideal of $A$ generated by $r+\operatorname{Nil}(R)$ can also be generated by some idempotent $f=e+\operatorname{Nil}(R)$, for some $e \in R$. Since Nil $(R)$ is a nil ideal, it follows from classical idempotent-lifting (see [10, Proposition 1, p.72]) that we may assume that $e$ is idempotent. In other words, $A(r+\operatorname{Nil}(R))=A(e+\operatorname{Nil}(R))$, with $e=e^{2} \in R$. In particular, $r \in R e+\operatorname{Nil}(R)$ and $e \in R r+\operatorname{Nil}(R)$. It follows that $e \in M_{1} \backslash \bigcup_{j=2}^{n} M_{j}$. Hence, $e \neq 0,1$. Therefore, $R e$ and $R(1-e)$ are each nonzero rings with identity and $R$ is the internal direct product of them: $R=R e \times R(1-e)$. Since $R$ is fragmented, it follows from Proposition 3.7 that $R e$ and $R(1-e)$ are each fragmented; moreover, each of these rings inherits zero-dimensionality from $R$. As $\operatorname{Spec}(R e)$ and $\operatorname{Spec}(R(1-e))$ are each nonempty and $\operatorname{Spec}(R) \cong \operatorname{Spec}(R e) \cup \operatorname{Spec}(R(1-e)), R e$ and $R(1-e)$ each have fewer than $n$ prime ideals. By the strong induction hypothesis, $R e$ and $R(1-e)$ are each von Neumann regular, and hence so is their product, $R$.

Although for zero-dimensional rings, reduced implies fragmented, our next example shows that the converse is false. Any such example is necessarily non-Noetherian by Corollary 4.4 (in fact, non-atomic by Proposition 3.11) and must possess an infinite number of maximal ideals (by Theorem 4.9).

EXAMPLE 4.10. There exists a zero-dimensional fragmented ring which contains a nonzero nilpotent element (and which is therefore not a von Neumann regular ring).

Proof: A ring $R$ with the asserted properties will be constructed as a direct limit (in fact, a directed union) of suitable rings $R_{n}$, as $n$ ranges over the positive integers. For simplicity, we begin with $R_{1}$ as the ring of dual numbers over the field $F_{2}$ with two elements, namely $R_{1}=\mathbf{F}_{2}[X] /\left(X^{2}\right)=\mathbf{F}_{2}[x]=\{0,1, x, 1+x\}$, where $x$ is the coset represented by $X$. Since $\operatorname{dim}\left(R_{1}\right)=0$, Theorem 3.5 shows that the obstacle to $R_{1}$ being fragmented (and the reason that $R_{1}$ is not fragmented) is that $x$ is not divisible in $R_{1}$ by a nonunit idempotent. Of course, $R_{1}$ can also be seen to be non-fragmented by Theorem 4.9 since $R_{1}$ contains a nonzero nilpotent, $x$.

The next step in the construction is to produce a zero-dimensional extension ring $R_{2}$ of $R_{1}$ in which each nonunit of $R_{1}$ (namely, $x$ ) has a nonunit idempotent factor in $R_{2}$. Let $Y_{1}$ be an indeterminate over $R_{1}$, and set $R_{2}:=R_{1}\left[Y_{1}\right] /\left(Y_{1}^{2}-Y_{1}, x Y_{1}-x\right)=R_{1}\left[y_{1}\right]$, where $y_{1}$ is the coset represented by $Y_{1}$. Note that $R_{2}$ is finite since it is an integral algebra-finite (hence module-finite) algebra over the finite $\operatorname{ring} R_{1}$. Hence, $\operatorname{dim}\left(R_{2}\right)=0$. We claim that $R_{1}$ is a subring of $R_{2}$, more precisely, that the canonical $R_{1}$-algebra homomorphism $R_{1} \rightarrow R_{2}$ is an injection. To prove this claim, one needs to observe that $R_{1} \cap\left(Y_{1}^{2}-Y_{1}, x Y_{1}-x\right)=0$. This, in turn, follows by considering the $R_{1}$-algebra homomorphism $R_{1}\left[Y_{1}\right] \rightarrow R_{1}$ determined by $Y_{1} \mapsto 1$, and so the claim has been established. Now, $x$ is divisible in $R_{2}$ by the idempotent $y_{1}$. Moreover, it can be seen that $y_{1}$ is a nonunit of $R_{2}$ by considering the $R_{1}$-algebra homomorphism $R_{1}\left[Y_{1}\right] \rightarrow R_{1}$ sending $Y_{1}$ to 
0 , and using the fact that $x$ is a nonunit of $R_{1}$.

We proceed with the inductive construction. Suppose that we have a chain $R_{1} \subseteq$ $R_{2} \subseteq \cdots \subseteq R_{n}$ of integral extensions of finite rings, for some $n \geqslant 2$, such that each nonunit of $R_{i}$ is divisible by some nonunit idempotent of $R_{i+1}$ whenever $1 \leqslant i \leqslant n-1$. Let $\left\{\alpha_{1}, \ldots, \alpha_{k}\right\}$ be the set of nonzero nonunits of $R_{n}$. Now, $R_{n+1}$ will be obtained by an inductive construction (within this induction step) resulting in a chain of rings

$$
R_{n}=A_{n, 0} \subseteq A_{n, 1} \subseteq \cdots \subseteq A_{n, k}=R_{n+1} .
$$

We obtain $A_{n, 1}$ from $A_{n, 0}=R_{n}$ just as $R_{2}$ was obtained from $R_{1}$, with $\alpha_{1}$ playing the earlier role of $x$. More precisely, let $A_{n, 1}=A_{n, 0}\left[Z_{1}\right] /\left(Z_{1}^{2}-Z_{1}, \alpha_{1} Z_{1}-\alpha_{1}\right)$. Exactly as above, we verify that $A_{n, 1}$ is a finite ring which contains and is integral over $A_{n, 0}$; and moreover that $\alpha_{1}$ is divisible in $A_{n, 1}$ by a nonunit idempotent (namely, the coset represented by $\left.Z_{1}\right)$. If $k \geqslant 2$, let $A_{n, 2}=A_{n, 1}\left[Z_{2}\right] /\left(Z_{2}^{2}-Z_{2}, \alpha_{2} Z_{2}-\alpha_{2}\right)$. Exactly as above, we verify that $A_{n, 2}$ is a finite ring which contains and is integral over $A_{n, 1}$; and moreover that $\alpha_{2}$ is divisible in $A_{n, 2}$ by a nonunit idempotent (namely, the coset represented by $Z_{2}$ ). Most importantly, $\alpha_{1}$ is also divisible in $A_{n, 2}$ by a nonunit idempotent because integrality ensures that nonunits of $A_{n, 1}$ remain nonunits of $A_{n, 2}$ (see [9, Theorem 44]). The above reasoning can be repeated essentially verbatim to carry out the induction step, thus producing $A_{n, 3}, \ldots, A_{n, k}=R_{n+1}$.

The above inductive construction produces a chain $R_{1} \subseteq R_{2} \subseteq \cdots \subseteq R_{n} \subseteq \cdots$ of finite (hence. zero-dimensional) rings such that for each $n, R_{n+1}$ is integral over $R_{n}$ and each nonzero nonunit of $R_{n}$ is divisible in $R_{n+1}$ by a nonunit idempotent. It remains only to show that $R:=\operatorname{dir} \lim R_{n}=\bigcup_{n=1}^{\infty} R_{n}$ has the desired properties. First, note that $\operatorname{dim}(R)=0$, since a standard result on direct limits [3, Exercice 11, p.VIII.82] ensures that $\operatorname{dim}(R) \leqslant \sup \operatorname{dim}\left(R_{n}\right)$. Next, to show that $R$ is fragmented, we employ the criterion from Theorem 3.5 as follows. If $y \in R \backslash U(R)$, choose $n$ such that $y \in R_{n} \backslash U\left(R_{n}\right)$, use the above construction to find a nonunit idempotent $e$ of $R_{n+1}$ which divides $y$ in $R_{n+1}$ (and hence divides $y$ in $R$ ), and observe that $e$ remains a nonunit in $R$ since $R$ is integral over $R_{n+1}$. Finally, $x$ is the desired nonzero nilpotent element of $R$.

\section{REFERENCES}

[1] D.D. Anderson and S. Valdes-Leon, 'Factorization in commutative rings with zero divisors', Rocky Mountain J. Math. 26 (1996), 439-480.

[2] N. Bourbaki, Commutative Algebra (Addison-Wesley, Reading, Mass., 1972).

[3] N. Bourbaki, Éléments de mathématique (chapitres 8-9), Algèbre Commutative (Masson, Paris, 1985).

[4] J. Coykendall and D.E. Dobbs, 'Fragmented domains have infinite Krull dimension', (submitted for publication).

[5] J. Coykendall, D.E. Dobbs and B. Mullins, 'On integral domains with no atoms', Comm. Algebra (to appear). 
[6] J. Coykendall, D.E. Dobbs and B. Mullins, 'Factorization in antimatter rings', in Advances in Commutative Ring Theory, Lecture Notes Pure Appl. Math. 205 (Dekker, New York, 1999), pp. 217-226.

[7] D.E. Dobbs, 'Fragmented integral domains', Portugal. Math. 43 (1985-1986), 463-473.

[8] J.A. Huckaba, Commutative rings with zero divisors (Dekker, New York, 1988).

[9] I. Kaplansky, Commutative Rings, (revised edition) (Polygonal Publishing House, Washington, N.J., 1994).

[10] J. Lambek, Lessons on rings and modules (Blaisdell, Waltham, MA, 1966).

[11] W.J. Lewis, 'The spectrum of a ring as a partially ordered set', J. Algebra 25 (1973), 419-434.

[12] R.S. Pierce, Modules over commutative regular rings, Memoirs Amer. Math. Soc. 70 (American Mathematical Society, Providence, R.I., 1967).

[13] O. Zariski and P. Samuel, Commutative algebra 1 (Van Nostrand, Princeton, 1958).

Department of Mathematics

North Dakota State University

Fargo ND 58105-5075

United States of America

Department of Mathematics and Statistics

Youngstown State University

Youngstown OH 44555-3302

United States of America
Department of Mathematics

University of Tennessee

Knoxville TN 37996-1300

United States of America 